\title{
An analysis of different sensors for turnout detection for train-borne localization systems
}

\author{
D. Stein, M. Lauer \& M. Spindler \\ Institute of Measurement and Control Systems, \\ Karlsruhe Institute of Technology, Germany
}

\begin{abstract}
Safe railway operation requires a reliable localization of trains in the railway network. Hence, this paper aims to improve the accuracy and reliability of train-borne localization systems proposed recently. Most of these approaches are based on a global navigation satellite system (GNSS) and odometers. However, these systems turned out to have severe shortcomings concerning accuracy and availability. We believe that the ability to detect turnouts and the branching direction thereon is the most valuable clue for improvement. Knowing the branching direction provides topological information about the train position. Thus, it complements the geographical information of GNSS and the longitudinal position information of odometers in an ideal way. With such a sensor setup a track-selective localization would be possible even if GNSS is unavailable or disturbed. Therefore, this paper compares the individual benefits of different sensor principles for turnout detection such as inertial measurement units (IMUs), cameras, and lidar (light detection and ranging) sensors. As a consequence, we focus on lidar sensors. For those we define requirements, review the market, and report the results of a case study in a tramway scenario. We proved that it is possible to detect rails, turnouts, and platforms. Finally we discuss our findings intensively and give an outlook on our further research.

Keywords: train-borne localization, turnout detection, sensor comparison, lidar sensor, GNSS, IMU, camera, eddy current sensor, digital track map.
\end{abstract}

\section{Introduction}

Infrastructure-based positioning systems as, e.g., described in [1] rely on a multitude of sensors along the track. Therefore, they induce high initial costs, 
require continuous maintenance, suffer from low accuracy and cause a standstill in the case of theft or vandalism [2]. Hence, recent localization approaches are based solely on train-borne sensors and are thus more flexible, especially when the tracks are not yet accordingly equipped.

In train control systems the reliable determination of the current train position in the railway network is the most relevant task. But, the effective accuracy of individual sensors for train-borne localization is poor. By combining different sensor principles and fusing their complementary information a higher accuracy could be achieved [2]. Most train-borne localization systems are thus based on global navigation satellite system (GNSS) receivers, odometers, and digital track maps (cf. fig. 1) [1,3-9]. The GNSS receiver provides the global position and the direction of the train. An odometer provides the distance covered along the track. Tachometers such as the eddy current sensor (ECS) $[5,9,10]$ and accelerometers as used, e.g., in inertial measurement units (IMUs) [3, 4, 6-8] are alternatives. The map contains information about the railway network, e.g., on geography, geometry, and topology.

However, those systems have severe shortcomings concerning accuracy and availability. Common odometers suffer from slip and slide between the wheel and track. IMUs tend to drift with increasing distance. Even promising alternatives such as Doppler radar sensors used in [3] or optical sensors like [11] may have problems in snowy and icy environments. The accuracy of GNSS receivers is also limited. Furthermore systematic errors of the GNSS position due to multipath effects and jammers, as well as the unavailability of GNSS signals in tunnels, deep valleys, and forests hamper precise localization. Those systems cannot guarantee a longitudinal position accuracy of up to $25 \mathrm{~cm}$ as stated, e.g., in [1]. Furthermore, they may not determine immediately on which of several parallel tracks the train is.

Regarding the structure of the railway network, turnouts enable the only possibility for trains to change the track. Hence, the detection of turnouts and the branching direction thereon is the most promising approach for accurate, reliable, and track-selective localization [12]. Knowing the branching direction provides topological information about the train position. This information complements the geographical position obtained from GNSS and the longitudinal position

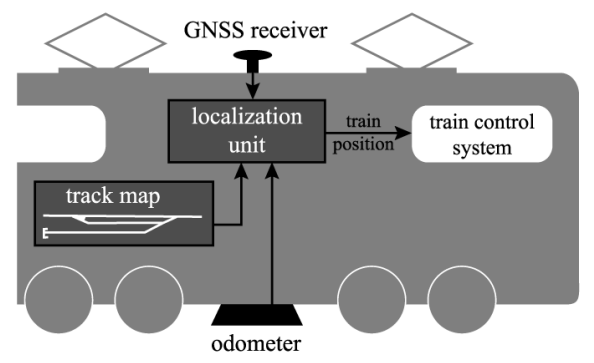

Figure 1: Common base setup for train-borne localization systems. 
derived from the odometer. Other landmarks that are proposed for position recalibration such as level crossings [7], bridges or tunnels [6] may support the localization in addition. But, they will not resolve ambiguities when passing turnouts and are thus not the most viable approach.

The remainder of this paper is organized as follows. Section 2 reviews different sensor principles for turnout detection. In sec. 3 we define requirements on lidar (light detection and ranging) sensors and review the market for those sensors, while sec. 4 describes a case study using a lidar sensor in a tramway scenario. Section 5 discusses the results of the case study and derives recommendations for an optimal usage of lidar sensors. Finally, sec. 6 summarizes the findings and gives an outlook on further research.

\section{Review of different sensor principles for turnout detection}

In this section we discuss different physical measurement principles that might be capable of detecting turnouts. Camera, ECS, IMU, and lidar sensors are the most promising sensors and considered here.

A camera mounted at the front of a train covers a large area in the driving direction of the train. The light that is reflected by a three-dimensional object, e.g., the rails and turnouts, creates a two-dimensional projection on a photosensor. Therefore, the results mainly depend on a homogeneous illumination of the environment. For interpreting the geometrical structure or the color of a surface image recognition is needed, but this requires high computational effort. Various weather conditions such as deep snow, heavy rain, fog, or shadows as well as different forms of the vegetation influence the measurement results. Using a second camera provides further information about the distance of an object and can improve the turnout detection as well. An alternative are time of flight (ToF) cameras. They also provide information about the three-dimensional structure of an object but with lower resolution.

In contrast, ECSs are less influenced by environmental conditions since they are based on the induction of eddy currents into the environment below the train, such as rails, rail clamps, and turnouts. The induced current emits an electromagnetic field which can be detected again. Different parts of a turnout, e.g., blade, frog and guard rails, can be distinguished by their characteristic signal sequence. Taking into account their chronological sequence the branching direction can be derived.

The lidar sensor is also an optical device. It measures the distance of an object while rotating a laser beam. Thereby, several hundred measurement points can be obtained in one or several layers. Those provide a height profile of the environment. Compared with cameras the lidar sensor is less influenced by the lighting or weather conditions due to the infrared laser that provides an adequate illumination. So, even in tunnels or under bridges appropriate measurements can be obtained.

An IMU delivers information about the relative position by measuring accelerations. The acceleration caused by turnouts might provide specific information on the branching direction. But, one cannot distinguish between a 


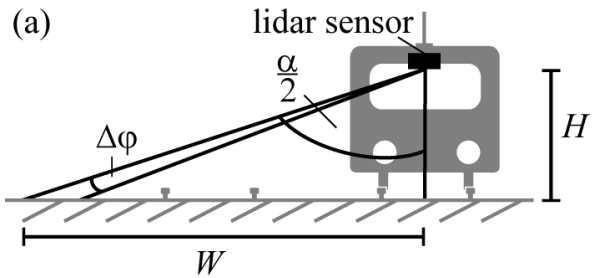

(b)

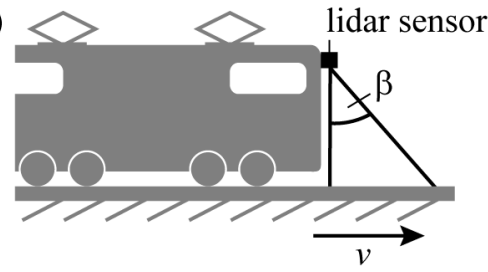

Figure 2: Single-layer lidar sensor on a train: (a) front view; (b) side view (width on ground $W$, height $H$, field of view $\alpha$, angular resolution $\Delta \varphi$, inclination $\beta$, velocity of the train $v$ ).

straight track and a straight main track within a turnout. The same applies to a curve on a normal track and within the diverted track of a turnout. Due to the measurement method, an IMU is influenced by vibrations of the train, sensor drift over time, and local gravity anomalies, but independent from weather conditions. In addition, the total error increases especially over long periods of time.

We conclude, that the lidar sensor which is capable of providing distance information of the environment directly is the best choice for our application. By emitting a laser beam in the infrared range, the sensor depends less on environmental conditions, such as lighting, shadows, or surface textures compared with cameras and is therefore preferred. The lidar sensor is also capable of detecting neighboring tracks. Since this a big advantage also compared with ECS and IMU sensors, lidar sensors will be analyzed in the next section in detail.

\section{Review of lidar sensors for detecting railway infrastructure}

The lidar sensor should detect parts of the railway infrastructure by measuring distances. Therefore, the working principle of lidar sensors, the size of the railway infrastructure elements, as well as possible mountings on a train have to be considered. We primarily focus on the detection of turnouts and will therefore also consider rails and tracks. First, we introduce the requirements that are relevant for this detection.

The most relevant lidar sensor properties are the angular resolution, field of view, measurement rate, range, and spot size [13]. Those will be described in detail assuming a single lateral scan layer as shown in fig. 2(a).

As the minimal distance between most tracks is $3.5 \mathrm{~m}$, the lidar sensor should cover at least this distance plus half of the standard gauge to the left and to the right side on straight tracks. Considering also the deflection of the train in curves the measurement width on ground $W$ should be at least $6 \mathrm{~m}$ to the left as well as to the right side [13]. Even when mounting the sensor on top of a train (height $H \approx 3.5 \mathrm{~m}$ ) the field of view has to be $\alpha \geq 120^{\circ}$ to reach $W=6 \mathrm{~m}$ (cf. fig. 2(a)). 


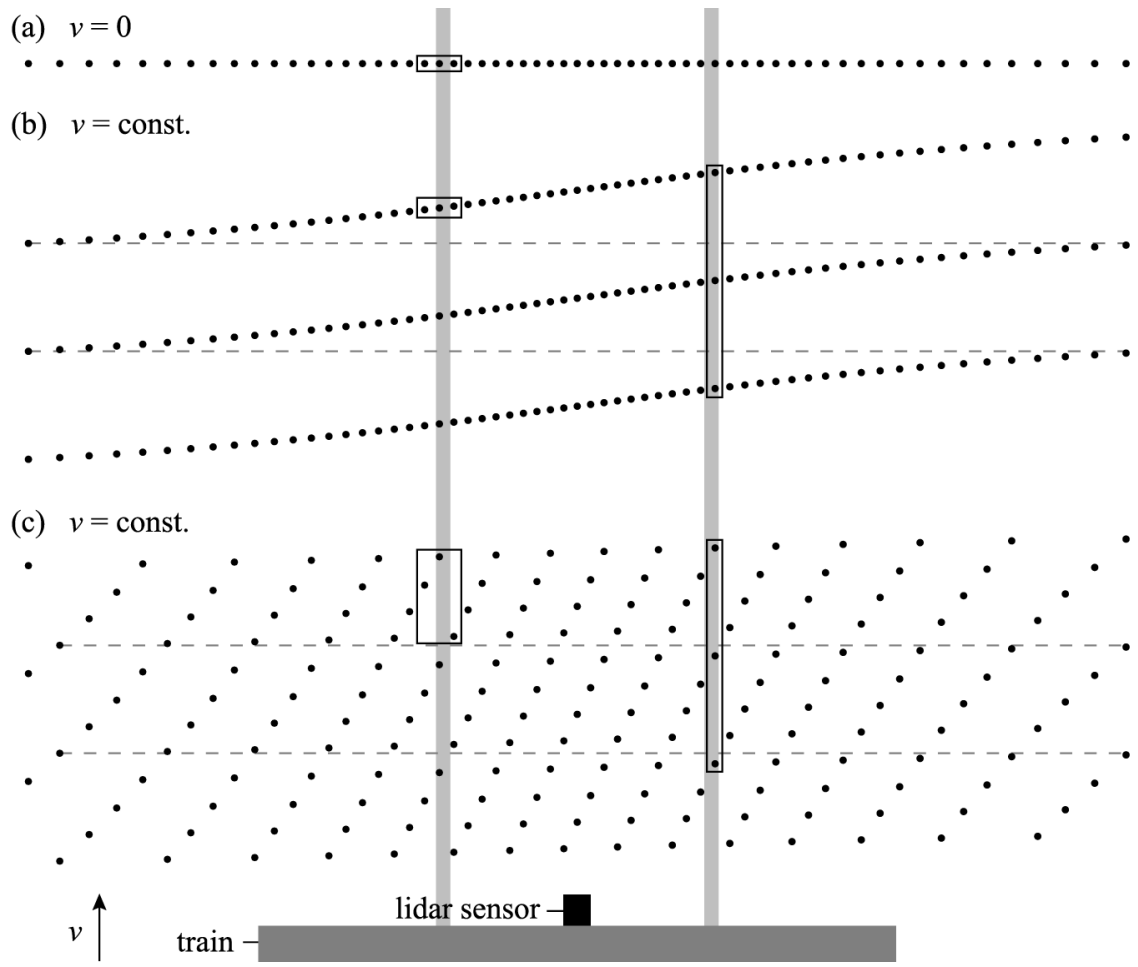

Figure 3: Straight track measured by a single lateral lidar scan layer (top view): (a) train stands still; (b) train moves with constant velocity $v$; (c) train moves with constant velocity $v$, but scans are interlaced and each scan is obtained in four rotations (boxes on the left rail mark the discretization in the lateral direction, boxes on the right rail in the longitudinal direction).

The range describes the measurement distance at which the sensor is capable of detecting objects. For our purpose distances up to $10 \mathrm{~m}$ are sufficient.

The most relevant property is the angular resolution $\Delta \varphi$ (cf. fig. 2(a)). It influences the discretization of objects in the lateral direction (cf. fig. 3(a)). For this purpose it should be as small as possible. To ensure a reliable detection of objects we claim that objects should be hit by at least three beams [14], especially as the train is moving. The smallest detectable object is determined by the sum of the spot size of the beam and the distance between those three beams, which should be as small as possible. Both properties scale with the distance. The former depends on the spot size at the outlet of the laser and the beam divergence, the latter depends on $\Delta \varphi$. In a height of $H=3.5 \mathrm{~m}$ with $\Delta \varphi=0.5^{\circ}$ and with negligible spot size the minimal object size at $W=6 \mathrm{~m}$ is $23 \mathrm{~cm}$. With $\Delta \varphi=0.1^{\circ}$ it is only $5 \mathrm{~cm}$, which allows, e.g., the profile of grooved rails on level crossings to be detected. 
Table 1: Primary requirements for the lidar sensors for detecting railway infrastructure.

\begin{tabular}{|l|c|c|}
\hline Property [unit] & Minimal value & Optimal value \\
\hline Angular resolution $\Delta \varphi\left[^{\circ}\right]$ & $<\frac{1}{2}$ & $<\frac{1}{8}$ \\
\hline Field of view $\alpha\left[^{\circ}\right]$ & $\geq 120$ & $\geq 180$ \\
\hline Measurement rate $f\left[\frac{\text { revolutions }}{\mathrm{S}}\right]$ & $\geq 30$ & $\geq 50$ \\
\hline Range [m] & \multicolumn{2}{|c|}{$0.2-10.0$} \\
\hline Layers & \multicolumn{2}{|c|}{$\geq 1$} \\
\hline Price [EUR] & $\leq 10000$ \\
\hline
\end{tabular}

Table 2: Features of the most promising single-layer lidar sensors for detecting railway infrastructure (field of view $\alpha$, angular resolution $\Delta \varphi$, measurement rate $f$ ) [15-18].

\begin{tabular}{|l|c|c|c|}
\hline Sensor & $\alpha\left[^{\circ}\right]$ & $\Delta \varphi\left[^{\circ}\right]$ & $f\left[\frac{\text { revol. }}{\mathrm{s}}\right]$ \\
\hline Hokuyo UTM-30LX-EW & 270 & 0.2500 & 40 \\
\hline Leuze rotoScan ROD4-58 plus & 190 & 0.3600 & 50 \\
\hline Pepperl+Fuchs OMD10M-R2000-B23-V1V1D & 360 & 0.0710 & 50 \\
\hline \multirow{2}{*}{ Sick LMS511-20100 PRO } & \multirow{2}{*}{190} & 0.1667 & 25 \\
\cline { 3 - 4 } & & 0.3300 & 50 \\
\hline
\end{tabular}

In addition to those properties that already influence the facilities of the lidar sensor when the train stands still, scans spread widely when the train has a nonzero velocity $v$ (cf. fig. 2(b)). The measurement rate $f$ determines the number of scans per second. The higher the measurement rate and the lower the train velocity is, the better objects are discretized along the track (cf. fig. 3(b)). For example, objects smaller than $2.75 \mathrm{~m}$ may be missed if we assume a rate of 30 scans per second while driving $100 \mathrm{~km} / \mathrm{h}$ and claiming that an object can only be recognized if it was hit by three scans. Note that the spaces between the measurement points get longer outwards in lateral direction due to the increasing radial distance from the lidar sensor (cf. fig. 3(a)). In contrast, spaces in the longitudinal direction remain constant since $v$ and $f$ are both constant (cf. fig. 3(b)).

One noteworthy thing is that some lidar sensors require several rotations to obtain one scan in higher resolution. In this interlaced mode spatially neighboring measurements are not measured one after the other. In particular, when objects or the sensor itself move, a typical zigzag pattern arises within a scan. Furthermore, spatially neighboring points are measured over a longer time period (cf. fig. 3(c)). 
Table 3: Features of the single-layer lidar sensor used for the case study (field of view $\alpha$, angular resolution $\Delta \varphi$, measurement rate $f$ ) [23].

\begin{tabular}{|l|l|l|l|}
\hline Sensor & $\alpha\left[^{\circ}\right]$ & $\Delta \varphi\left[^{\circ}\right]$ & $f\left[\frac{\text { revolutions }}{\mathrm{S}}\right]$ \\
\hline \multirow{2}{*}{ Sick LMS200-30106 } & 100 & 0.25 & 18.75 \\
\cline { 2 - 4 } & 180 & 0.50 & 37.50 \\
\hline
\end{tabular}

Our requirements on lidar sensors are summarized in Table 1. We found four vendors that provide single-layer sensors which meet most requirements (cf. tab. 2). If a vendor offers several sensors, we selected the most promising one. Due to our restrictions on angular resolution and measurement rate multi-layer sensors used for driver assistance (e.g. ibeo LUX [19]) and autonomous driving (e.g. Velodyne lidar [20]) have not been surveyed. Also high-end sensors already established in the railway domain (e.g. Riegl VQ-450 [21] or High Speed Profiler of Fraunhofer Institute for Physical Measurement Techniques [22]) are usually ten times more expensive than the price level admissible for a train localization system.

Based on our theoretical findings, the Pepperl+Fuchs lidar sensor is the most appropriate device since it has by far the finest angular resolution and has the highest measurement rate. Alternatives are the sensors from Hokuyo and Sick. The former one allows quarter degree scans and is also quite fast. The latter one offers up to six measurements per degree.

\section{Case study of a lidar sensor in a tramway scenario}

For a case study we used the lidar sensor described in tab. 3 and performed test drives in a tramway scenario in the city of Karlsruhe. Thereto, we mounted the sensor at the front of a road vehicle. So we were able to validate our concept without requiring a special train and avoided the respective expenses. Furthermore, we could test several configurations (e.g. heights $0.3 \mathrm{~m} \leq H \leq 1.0 \mathrm{~m}$ and inclinations $0^{\circ} \leq \beta \leq 90^{\circ}$, cf. fig. 2) even before purchasing a sensor advised in sec. 3.

Figure 4(a) shows a series of about 250 scans while passing a turnout trailing. Motivated by $[7,14]$ we used a template matching approach that detected grooved rails in the lidar sensor measurements. Therefore, we required that each groove is hit by at least three beams in the lateral as well as in longitudinal direction. In each scan we tested whether the measurements are in a local minimum, since the grooves are located below their environment. To distinguish a groove from uneven road we considered a minimal groove depth. Our approach detected the rails marked in the lateral cut in fig. 4(b). We limited therein the detection area to $X= \pm 1.2 \mathrm{~m}$ to cover at least our track and considered thereby deflections of the vehicle. Furthermore, fig. 4 (b) shows that the blade $(X \approx \pm 0.8 \mathrm{~m})$ is 
(a)

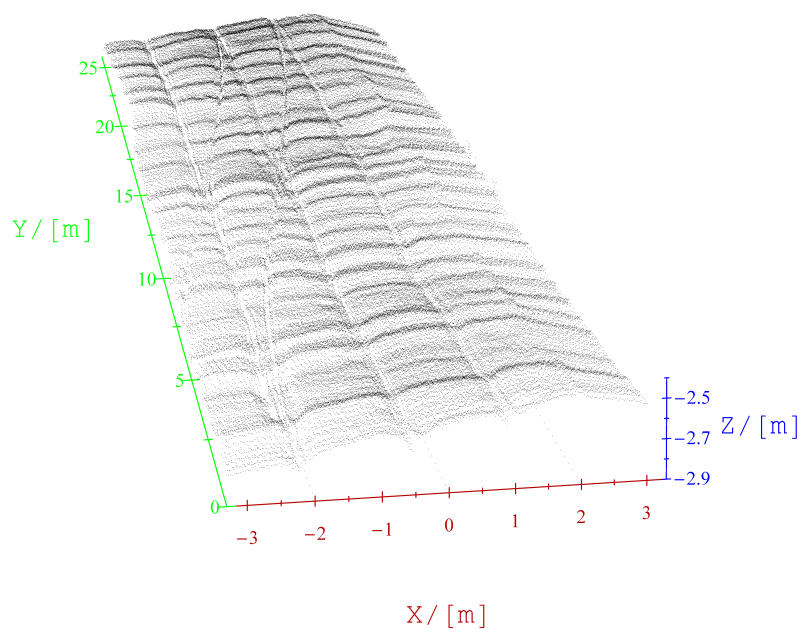

(b)

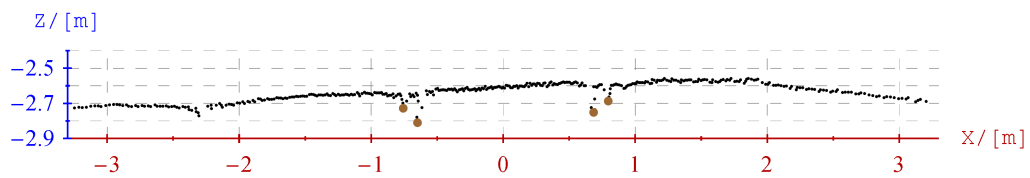

(c)

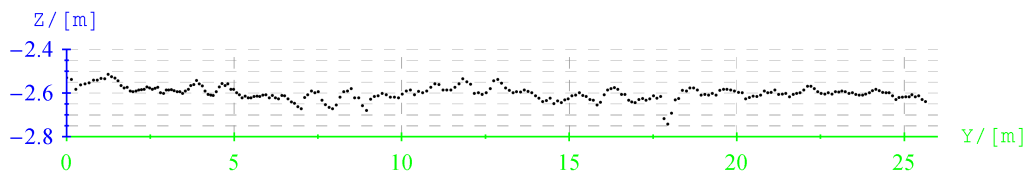

Figure 4: Lidar sensor scans when passing a turnout with grooved rails and standard gauge trailing: (a) 3D view, (b) lateral cut $(z=23.7 \mathrm{~m}$, detected rails are marked with brown circles), (c) longitudinal cut $(x=0 \mathrm{~m})$ (height $H=1 \mathrm{~m}$, inclination $\beta=68^{\circ}$, field of view $\alpha=100^{\circ}$, angular resolution $\Delta \varphi=0.25^{\circ}$, velocity of the vehicle $\bar{v}=2 \mathrm{~m} / \mathrm{s}$ ).

distinctive, since it is deeper than ordinary grooved rails $(X=-2.3 \mathrm{~m})$. In addition, converging and diverging pairs of rails with a similar gauge could be used to detect turnouts. Without the restrictions mentioned before we could even detect the single rail on the left side, but would not be able to cross-check the detection by comparing the gauge.

Figure 5(a) shows another series of about 130 scans obtained with the same setup. One can see that besides the already detected grooved rails laminar objects such as curbs or platforms on the right side also have a distinctive profile (cf. also fig. 5(b)). They could be detected either by matching their exact profile or by considering their difference in height as well as their distance from the track. Due 
(a)

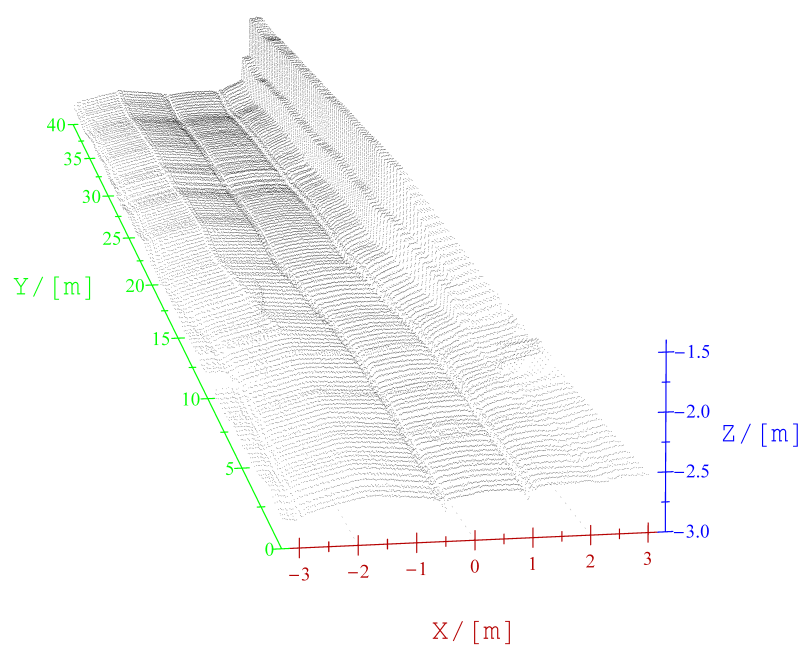

(b)

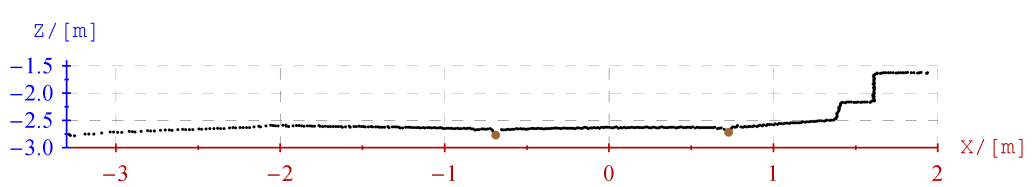

Figure 5: Lidar sensor scans when passing a tramway stop with grooved rails and standard gauge: (a) 3D view, (b) lateral cut $(z=35.5 \mathrm{~m}$, detected rails are marked with brown circles) (height $H=1 \mathrm{~m}$, inclination $\beta=68^{\circ}$, field of view $\alpha=100^{\circ}$, angular resolution $\Delta \varphi=0.25^{\circ}$, velocity of the vehicle $\bar{v}=5.7 \mathrm{~m} / \mathrm{s}$ ).

to their dimensions those objects are detectable even where grooved rails are not, since the minimal object size becomes too large.

The results of this case study prove that we are able to detect rails in a tramway scenario using a lidar sensor. This is noticeable since the recognition of grooved rails (width of groove about $6 \mathrm{~cm}$ ) is more difficult compared with that of Vignoles rails (height at least $13 \mathrm{~cm}$ ). Furthermore, we proposed additions to detect also turnouts and platforms, which have their distinctive properties, too.

\section{Discussion and recommendations for the sensor configuration}

In Section 4 we demonstrated that lidar sensors are appropriate for turnout detection. Thus, we will discuss the influence of the mounting (cf. fig. 2) and further sensor characteristics on the measurement quality. Furthermore, we derive recommendations for an optimal lidar sensor configuration from our practical experiences, whereas Table 1 summarizes the optimal values of the lidar sensor properties. 
The optimal height $H$ equals the desired width on ground $W$, since the measured distance $D=\sqrt{W^{2}+H^{2}}$ is minimal then. Due to practical reasons in most cases the inclination will be $\beta \gg 0^{\circ}$, since a mounting at, e.g., $H=6 \mathrm{~m}$ is not feasible (cf. Section 4). However, lidar sensors should measure surfaces orthogonally, since the surface orientation and the surface reflectivity influence the measurement quality. With increasing inclination, the distance, the corresponding error, and the risk of undesired reflections increase. In addition, vibrations and the pitching of the train shift the scan layer and therefore influence the measurements. This can be seen in the longitudinal cut of the scans in fig. 4(c) with $D=2.6 \pm 0.1 \mathrm{~m}$. Hence, the inclination should be as small as possible. In contrast, the measured groove depth increases by $(\cos \beta)^{-1}$, such that grooved rails become more significant compared with the environment. All in all, we recommend to choose the smallest inclination.

By mounting the sensor off-centered and yawing it the discretization in lateral direction can be improved. However, another sensor on the opposite side is needed. And a combination of multiple sensors in different layers is more flexible than using one multi-layer sensor [13].

When a beam partially strikes the edge of an object, it is unpredictable whether the distance of the object or the background will be measured. To capture those values, the detection of multiple echoes is desirable. And as done in [7], to distinguish between different materials a concurrent measurement of distance and remission is appreciated. Since both features are not supported by the sensor used for our test drives, we were not yet able to test them.

As shown in fig. 3(c) the use of several rotations to obtain one scan has an influence on the spatial and temporal neighborhood of the measurements. Contrary to our expectation the zigzag pattern was not dominant in the test drives, although the sensor required four rotations per scan and our vehicle was not guided. If this sensor behavior cannot be avoided and a high resolution is needed, the scans could be split into partial scans for each rotation or one ignores the volatile neighborhood as drawn, e.g., in fig. 4(a).

\section{Conclusions and future work}

Current train-borne localization systems using GNSS, odometers, and digital track maps have severe shortcomings concerning accuracy and reliability. Thus, they cannot always determine immediately on which of several parallel tracks the train is located. Although, this is the most important prerequisite for the safety of train control systems. Therefore, we motivated the necessity of a turnout detector and identified lidar sensors as the most promising choice to complement those systems.

Our main contributions of this paper are the identification of requirements on lidar sensor properties and the recommendation of an adequate setup. Furthermore, we practically evaluated their ability for railway infrastructure detection in several test drives in a tramway scenario. The paper is thus based on a systematic review of lidar sensors. Thereby, angular resolution, spot size, and measurement 
rate has to be noted as the most relevant sensor properties. They influence the discretization and as a consequence the detection of objects. In several test drives we demonstrated the ability to recognize grooved rails although their characteristics are harder to detect compared with those of Vignoles rails. By combining this information with the distinctive properties of the blade as well as the knowledge on converging or diverging tracks we provide a basis for the detection of turnouts and the branching direction thereon.

All in all, it is worthwhile to focus furthermore on the robust detection of railway infrastructure elements such as turnouts and neighboring tracks. Thereby we will consider the different characteristics of tracks on grooved rails as well as on Vignoles rails. We are confident of detecting also the branching direction on turnouts and plan to evaluate the proposed Pepperl+Fuchs as well as the latest Sick sensor therefor. For evaluating the performance of the lidar sensor even in more complicated situations we envisage tests under rainy, snowy, and foggy weather.

\section{Acknowledgements}

This work was partially supported by the European GNSS Agency (GSA) within the FP7 project GaLoROI, project No. 277698-2. The authors would also thank Z. M. Pólya for their extraordinary commitment during her internship and B. J. Riehm for preparing the test drives.

\section{References}

[1] Albrecht, T., Lüddecke, K. \& Zimmermann, J., A precise and reliable train positioning system and its use for automation of train operation. Proc. IEEE Int. Conf. on Intell. Rail Transp., pp. 134-139, 2013.

[2] Mirabadi, A., Mort, N. \& Schmid, F., Application of sensor fusion to railway systems. Proc. IEEE/SICE/RSJ Int. Conf. on Multisensor Fusion and Integration for Intell. Syst., pp. 185-192, 1996.

[3] Albanese, A., Labbiento, G., Marradi, L. \& Venturi, G., The RUNE project: The integrity performances of GNSS-based railway user navigation equipment. Proc. Joint Railway Conf., pp. 211-218, 2005.

[4] Jiang, Z., Digital Route Model Aided Integrated Satellite Navigation and Low-cost Inertial Sensors for High-Performance Positioning on the Railways. Ph.D. thesis, University College London, 2010.

[5] Hensel, S., Hasberg, C. \& Stiller, C., Probabilistic rail vehicle localization with eddy current sensors in topological maps. IEEE Trans of Intell. Transp. Syst., 12(4), pp. 1525-1536, 2011.

[6] Rahmig, C., Lüddecke, K. \& Lemmer, K., Tunnels and bridges as observable landmarks within a modified multi-hypothesis based mapmatching algorithm for train positioning. Proc. European Navigation Conf., 2012. 
[7] Rahmig, C., Johannes, L. \& Lüddecke, K., Detecting track events with a laser scanner for using within a modified multi-hypothesis based map-matching algorithm for train positioning. Proc. European Navigation Conf., 2013.

[8] Heirich, O., Robertson, P. \& Strang, T., RailSLAM - localization of rail vehicles and mapping of geometric railway tracks. Proc. IEEE Int. Conf. on Robotics and Automation, pp. 5192-5199, 2013.

[9] Lauer, M. \& Stein, D., Algorithms and concepts for an onboard train localization system for safety-relevant services. Proc. IEEE Int. Conf. on Intell. Rail Transp., pp. 65-70, 2013.

[10] Engelberg, T. \& Mesch, F., Eddy current sensor system for non-contact speed and distance measurement of rail vehicles. Computers in Railways VII, eds. J. Allan, R. Hill, C. Brebbia, G. Sciutto \& S. Sone, WIT Press: Southampton, pp. 1261-1270, 2000.

[11] HaslerRail AG, CORRail 1000, 2012. Product description available online at http://haslerrail.com.

[12] Mesch, F., Puente León, F. \& Engelberg, T., Train-based location by detecting rail switches. Computers in Railways VII, eds. J. Allan, R. Hill, C. Brebbia, G. Sciutto \& S. Sone, WIT Press: Southampton, pp. 1251-1260, 2000.

[13] Pólya, Z.M., Aufbau und Maße im Gleisnetz und Funktionsweise Laserscanner (structure and dimensions of the railway network and operating principle of laser range measurements), 2013.

[14] Blug, A., Baulig, C., Wölfelschneider, H. \& Höfler, H., Fast fiber coupled clearance profile scanner using real time 3D data processing with automatic rail detection. Proc. IEEE Intell. Veh. Symp., pp. 658-663, 2004.

[15] Hokyuo Automatic Co., UTM-30LX-EW, 2012. Product description available online at http://hokuyo-aut.jp.

[16] Leuze electronic GmbH + Co. KG, rotoScan ROD4 plus, 2013. Product description available online at http://leuze.de.

[17] Pepperl+Fuchs Group, OMD10M-R2000-B23-V1V1D, 2013. Product description available online at http://pepperl-fuchs.com.

[18] SICK AG, Laser Measurement Sensors of the LMS5xx Product Family, 2012. Operating instructions available online at https://www.mysick.com.

[19] ibeo Automotive Systems GmbH, ibeo LUX, 2013. Product description available online at http://ibeo-as.com.

[20] Velodyne Lidar Inc., High Definition Lidar HDL-64E S2, 2010. Product description available online at http://velodynelidar.com.

[21] RIEGL Laser Measurement Systems GmbH, VQ-450, 2012. Product description available online at http://riegl.com.

[22] Fraunhofer Institute for Physical Measurement Techniques IPM, High Speed Profiler (HSP), 2013. Product description available online at http://www.ipm.fraunhofer.de.

[23] SICK AG, LMS 200 / LMS 211 / LMS 220 / LMS 221 / LMS 291 Laser Measurement Systems, 2003. Technical description available online at http://sick-automation.ru. 